\title{
Integrating Experimental Archaeology, Phytolith Analysis and Ethnographic Fieldwork to Study the Origin of Farming in China
}

\author{
Tracey L.-D. Lu
}

\begin{abstract}
Professor Peter Bellwood is a leading scholar studying the global origin and expansion of farming, and has published extensively on this topic (e.g. Bellwood 1978, 2005). With his guidance, the author has studied the origins of agriculture in mainland China since the 1990s, and has pursued a number of different archaeological and experimental methods to try and tackle the issue since then. In this chapter, I outline some of the methods of experimental archaeology that have been applied to determine the nature of the origins and development of prehistoric agriculture onwards, summarising the outcome of experimental archaeology integrated with phytolith analysis and ethnographic fieldwork conducted. I argue that integrating cultivation experiments, use-wear analysis of tools, phytolith analysis and ethnographic information can provide novel data and inspirational ideas within the context of the natural and cultural changes involved in and caused by cultivation. Remaining questions for further study are also discussed.
\end{abstract}

\section{Introduction}

Experiments have been used in archaeological studies since the nineteenth century in Europe (Coles 1979; Forrest 2008). Early inquiries focused on the functional analysis of ancient tools, but human behaviour, processes and social systems have become the objectives of experimental archaeology since the 1960s in order to 'generate and test hypotheses to provide or enhance analogies for archaeological interpretation' (Mathieu 2002: 1), providing a much broader scope for experimental archaeology.

Cultivation experimentation is an approach within experimental archaeology. Clearly, cultivation is a crucial process for the domestication of wild plants. Generally speaking, cultivation refers to a cultivation process from broadcasting seeds or planting, to field management including watering, fertilising and weeding, to harvesting, and selecting seeds for the following years. This is a dynamic, continuous and diversified process between humans and nature, particularly in terms of plants and their environment. It is in this process that the mutations of wild plants have been selected, fixed and reproduced, and wild plants have gradually been domesticated.

1 Tracey Lu died on 21 March 2016 in Melbourne, Australia. 
Cultivation experimentation is a pre-designed and controlled process of replicating or modelling cultivation activities for ascertained objectives. Agronomists and other scholars have used this approach since at least the early twentieth century for various purposes, ranging from increasing productivity, to producing hybrids, and assessing the impact of modern cultivation techniques on wild plants. In the 1950s, Oka and Chang (1959) conducted cultivation experiments using wild rice, and discovered that its biological characteristics could change rapidly through cultivation. However, they used relatively modern agricultural techniques such as transplanting and irrigation, and their objective was to tackle an agronomical issue instead of the archaeological issue of the origin of rice farming.

In the 1960s, some archaeologists began to use harvesting and cultivation experiments to study the origin and development of prehistoric agriculture in the Middle East. Jack Harlan conducted a pioneering wild wheat harvesting experiment in Turkey, through which he obtained novel and quantitative data for productivity. Based on this, he argued that the high productivity of wild wheat might have been a major impetus for the origin of wheat cultivation and eventually wheat domestication in the Middle East (Harlan 1967). Harvesting and systematic cultivation of wheat and barley, as well as associated pollen, use-wear analysis and ethnographic studies have been carried out by many scholars since the 1960s (Anderson 1992; Hillman and Davies 1992). Results from these cultivation experiments have illustrated that they can provide important data on many issues, including the productivity of wild and domesticated plants, the impact of different agricultural techniques, domestication rates, and the dynamics between cultivation, human cultural change and the impact upon the environment.

Inspired by the aforementioned works, cultivation experiments using green foxtail millet (Setaria viridis P. Beauv) and its domesticated counterpart foxtail millet (Setaria sativa), and perennial wild rice (Oryza rufipogon Griff.) and domesticated rice (Oryza sativa) were carried out from 1999 to 2005 and in 2012 in the middle Yellow River Valley and South China respectively (Lu 2002, 2013), associated with phytolith analysis, use-wear analysis and ethnographic studies. This paper summarises the major outcome of these works and discusses outstanding questions.

\section{Objectives}

Cultivation experiments with phytolith and use-wear analysis, and ethnographic studies constitute an integrated approach, intended to provide a multi-perspective examination of the functions of prehistoric tools, human behaviour, and the processual and other systematic changes to ancient foragers' and farmers' cultures associated with plant and other environmental alterations. The objectives of this integrated approach are as follows:

1. To understand the biological differences between wild and cultivated plants, and the impact of human cultivation on the evolutionary process from wild to domesticated plants, particularly on how water control, soil fertilisation, harvesting, seed selection and other cultivation behaviour might have selected, concentrated and reproduced wild plants with mutated genotypes, and also changed the growth cycle, productivity and reproduction of these plants, and consequently facilitated the domestication process.

2. To understand the impact of cultivation activities on human society and culture, in aspects such as cultivation and the occurrence and extension of sedentism, farming and settlement planning, the development of farming land as immovable assets, and the emergence of land ownership and the notion of private property. 
3. To understand the impact of different cultivation activities on the environment, including the flora and fauna. Various agricultural techniques might have been used at different periods of time in various regions, such as the slash-and-burn method used for dry-land farming, and the fish-agriculture symbiosis in wet-rice paddy fields. Have these activities caused deforestation, land erosion and loss of soil nutrition, have they decreased water supply and reduced floral and faunal diversity and quantity, and have they altered the landscape, all of which have eventually produced significant, indeed irreversible impacts on human society and culture from the past to the present? These are important questions for archaeology.

The ultimate goal of this integrated approach is to obtain novel qualitative and quantitative data in order to understand the economic, social and ideological changes that occurred in forager and farmer lifeways in antiquity, to tackle the questions of why, how, and by whom agriculture originated and developed in mainland China, and what were the consequences of this prehistoric cultural change.

\section{Methods}

The integrated approach of the present study chose to apply methods from agronomy, palaeoethnobotany and ethnology based on their suitability for the research questions and objectives, considering also the land, water, plant seed and human resources available for the study. Data collected from cultivation experiments, use-wear analysis, and the experiences of contemporary rice and millet farmers have been used to confirm or falsify relevant hypotheses, such as lack of cultivation management as a cause for low or nil crop returns. Research findings from agronomists and biologists have also been incorporated into a background literature review.

Briefly, the following projects have been carried out:

1. Cultivation experiments on green foxtail and perennial wild rice using the most 'primitive' techniques have been conducted. Based on ethnographic data from Taiwan Aborigines, a cultivation experiment with slash-and-burn planting and harvesting green foxtail was conducted in North China in 1999-2001 (Figure 14.1) (Lu 2002). Wild-rice cultivation using seed broadcasting and water maintenance (Figure 14.2) without fertiliser or controlled irrigation was carried out from 2000 to 2005 (Lu 2013), and a comparative cultivation experiment on wild and domesticated rice was conducted in Hong Kong in 2012 (Figures 14.3 and 14.4).

2. Harvesting wild and domesticated foxtail millet and rice was conducted from 1996 to 2002 in the Yellow River Valley and South China respectively using a variety of methods to provide comparative data for the productivity of wild and domesticated plants (Lu 1998, 2006). Tools replicating Rtools of prehistoric stone flakes, ground stone sickles, bone and shell knives (Figure 14.5) were used for the harvest in order to test the efficiency of these prehistoric implements. Additionally, they were observed under a microscope in order to establish reference data for use-wear and residue analysis of prehistoric implements found in South China and Hong Kong.

3. Soil samples were collected from slash-and-burn green foxtail fields and from foxtail millet fields involving modern farming methods. The phytolith and charcoal assemblages were compared in 1999 in order to study the impact of cultivation upon soil composition and to provide reference data to assist in the search for prehistoric farmlands in China (Figures 14.6 and 14.7). 
4. Ethnographic studies of contemporary rice farmers in South China and foxtail millet farmers in the middle Yellow River Valley and Taiwan have been carried out since late 1999 in order to collect data on cultivation techniques, crop productivity and the significance of rice and millet in farming societies.

5. Verbal and visual recording has been carried out during all of the aforementioned projects. The records cover daily climate, the amount of seeds broadcast and harvested, methods of cultivation and harvesting, time, labour and the materials used for manufacturing replicas, etc.

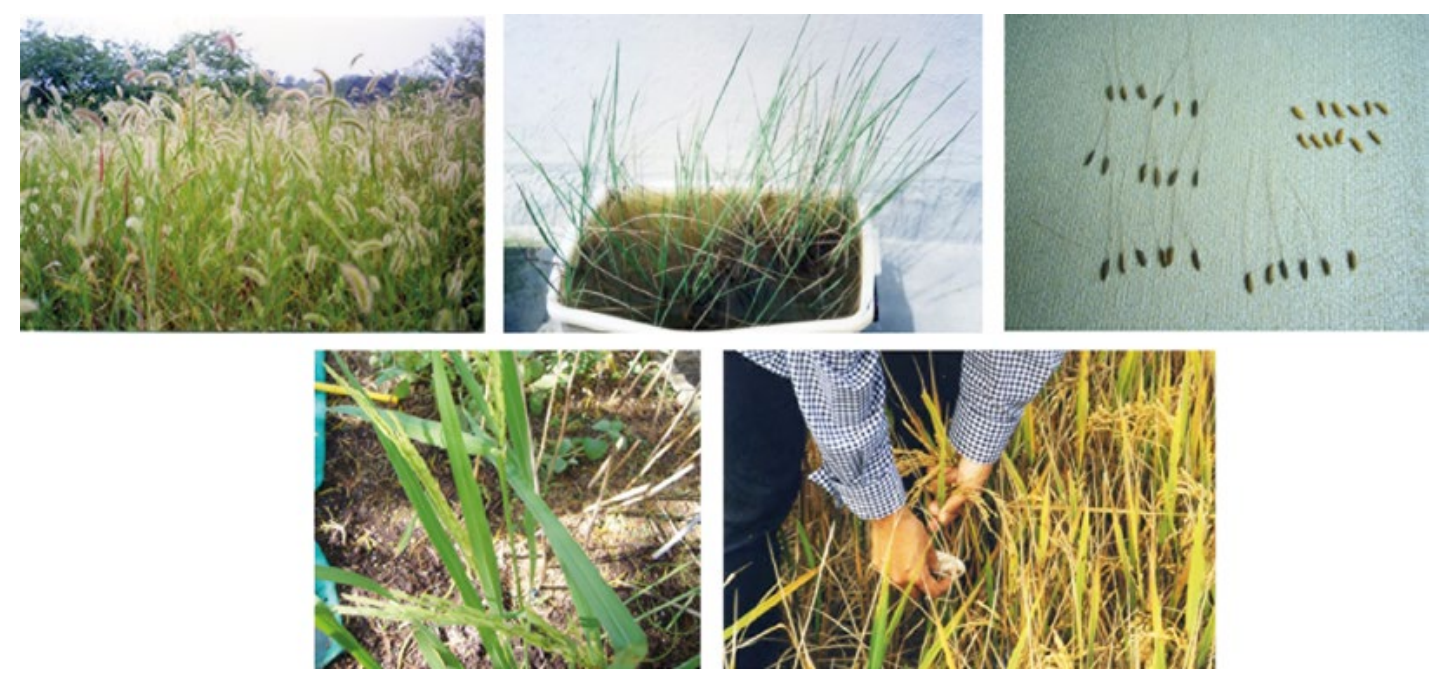

Figure 14.1 Cultivated green foxtail in the loess in North China (top left).

Source: Photo taken by the author in 1999.

Figure 14.2 Cultivated perennial wild rice in South China (top middle).

Source: Photo taken by the author in 2002.

Figure 14.3 Wild perennial rice (Oryza rufipogon) and domesticated rice (Oryza sativa) grains for the comparative cultivation experiment in 2012 (top right).

Source: Photo taken by the author in 2012.

Figure 14.4 Flowering domesticated rice (bottom left).

Source: Photo taken by the author in 2012.

Figure 14.5 Harvesting domesticated rice using shell knife (bottom right).

Source: Photo taken by Fu Xiaguo in 2000.

Various criteria have been used to control the cultivation experiments. For example, as the objective for perennial wild rice was to know the germination rate, time and productivity of the plant at the initiation of rice cultivation, the experiment did not use water control, fertiliser or other farming techniques. The cultivation of green foxtail also simulated an 'initial farming' process, and neither irrigation nor fertilising were applied ( $\mathrm{Lu} 2002)$. The timing and methods of harvesting have also been determined by the research objectives (Lu 2002, 2006, 2013).

A detailed example is provided by the comparative cultivation, without fertiliser or irrigation, of wild and domesticated rice in 2012, in order to compare their productivity (grain output compared to grain input). Grains of perennial wild rice (Oryza rufipogon Griff.) and domesticated rice (Oryza sativa) were selected and broadcast in boxes or basins with a relatively stable amount of water. While it is essential to supply or drain out water at certain times in contemporary rice 
farming in order to raise productivity, these complex water control techniques were not applied in the cultivation experiment, as it requires an irrigation mechanism, which may not have been available at the beginning of rice cultivation. Results show that the domesticated rice cultivated by this 'primitive' approach could still geminate, flower and ripen (Figure 14.4), but none of the 24 perennial wild rice grains germinated. Apart from confirming the zero or negative productivity when attempting to cultivate wild rice without irrigation, the results also demonstrate the critical importance of the genotypic differences between wild and domesticated rice.

\section{Findings}

The findings from the above projects can be summarised as follows:

1. The productivity of green foxtail in its first year of cultivation is over 9.38, while the productivity of perennial wild rice in its first year of cultivation is negative 20 (or worse), as only four grains of wild rice were harvested in the autumn after 80 grains were broadcast in the spring of 1999, and no grains were harvested from the 24 grains cultivated in 2012 (Lu 2002, 2013).

- One of the main causal factors of this huge difference is the biological difference between green foxtail as an annual grass reproducing itself by producing large amounts of seed, and wild rice as a perennial plant reproducing itself mainly by ratooning.

- Amongst all of the progenitors of domesticated crops in the world, wild rice is the only potential perennial (the issue of whether perennial or annual wild rice is the direct progenitor of domesticated rice is still a question under debate). The comparative cultivation experiments using green foxtail and perennial wild rice illustrate that the return on cultivating the latter is extremely low, even negative, thus highlighting the question of whether the cultivation of wild rice originated for the purpose of increasing food supplies or for other purposes. Hayden (2003) has argued that wild rice might have been a luxury food in prehistory, used for ritual purposes. Further archaeological studies are required to address this issue.

2. It has been observed that the stem diameter of both foxtail millet and domesticated rice is significantly bigger than their wild counterparts (Lu 1998, 2002, 2006, 2013), which might have facilitated more efficient transforming and greater utilisation of nutritional elements from the soil. In other words, the evolution from wild to domesticated plants involved changes to the whole plant, not just the spikelet base. Thus it is important for archaeologists to collect and study every fragment of rice and millet, not just the spikelets, found in archaeological deposits in order to holistically study the domestication of plants and the origin and development of prehistoric agriculture.

3. The result of the comparative cultivation experiments of perennial wild rice (Oryza rufipogon) and domesticated rice (Oryza sativa) conducted in 2012 illustrates that biological characters determine the growth cycle and productivity of the two species. However, according to contemporary farmers, both foxtail millet and domesticated rice require regular management in their watering, fertilising and weeding, otherwise the productivity could become extremely low or even negative. For example, a millet farmer in the middle Yellow Valley told the author in 1999 that over 90 per cent of the foxtail millet he had planted died out in that year because he did not have time to look after the crop. Thus, it can be argued that when prehistoric people began to cultivate green foxtail or wild rice, similar management must have been necessary, which would have demanded a sedentary lifestyle of the cultivators. Based on archaeological data in mainland China to date, sedentism occurred after the occurrence of farming, and the need to manage farming lands must have played an important role. 
4. There are similarities and differences in terms of cultivation techniques and crop management for green foxtail and wild rice. While the former can be cultivated on any piece of land, the latter must be cultivated in a field with constant water supply. It is hypothesised that the prehistoric farmers might have used small water ponds or land along rivers/streams to cultivate wild rice at the initial stage, but eventually prehistoric farmers had to construct paddy fields with banks to control the water level, without which it would have been

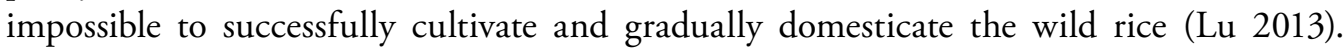
Archaeologists have found rice paddy fields in the middle and lower Yangzi River Valley, the earliest dated to approximately 7,000 years ago (Zheng et al. 2009). As the creation of rice fields required labour input, and rice, millet and other crops could be produced continuously from farmed fields, fields eventually became the immovable property of farmers, who had to become more sedentary in order to ensure their access to land and manage and protect their crops. The continuous use of farming land as a productive asset must have led to formal land ownership in ancient China, and the protective walls of the Neolithic town Chengtoushan dated to approximately 6,000 years ago in the middle Yangzi River Valley might have served the need of protecting rice farming land ( $\mathrm{Lu} 2013$ ).

5. The slash-and-burn farming technique adds many charcoal grains to the soil, associated with large quantities of phytoliths from the grass family (Figures 14.6 and 14.7). This could be a useful reference point for identifying prehistoric farming land. Soil analysis has been used to identify prehistoric rice paddy fields and farming techniques in the Yangzi River Valley (e.g. Zheng et al. 2009; Hu et al. 2013), but the farming lands of millets and other crops are yet to be identified. Though the absence of diagnostic millet phytoliths remains a major obstacle in this issue, the presence of a large amount of charcoal and phytolith from Paniceae could be a useful indicator.

6. Use-wear analysis of tools used in harvesting experiments indicates that cutting grasses does produce unique use-wear patterns, but more studies are required in order to distinguish whether the implements were used for cutting crops or grasses.

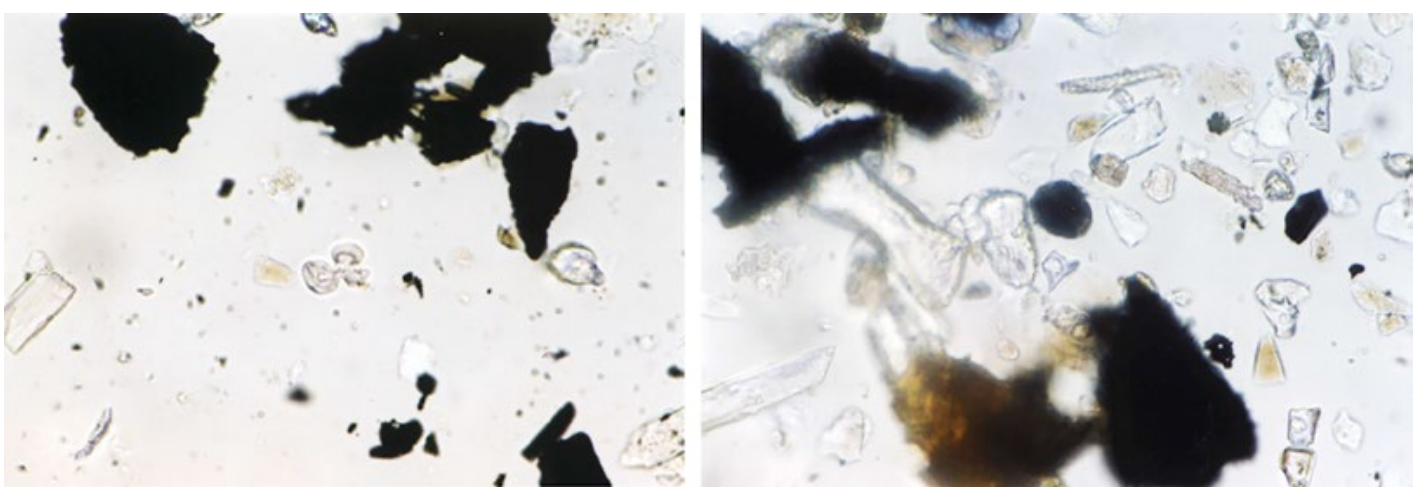

Figure 14.6 Charcoal and phytolith grains found in contemporary farming land of foxtail millet. Magnification about 400X (left).

Source: Micro-photo taken by the author in 1999.

Figure 14.7 Charcoal and phytolith grains found in contemporary farming land of green foxtail. Magnification about 400X (right).

Source: Micro-photo taken by the author in 1999. 


\section{Conclusion}

The projects summarised here indicate that integrating cultivation experiments, phytolith and use-wear analyses and ethnographic studies can provide novel and useful data for studying the origin and development of farming and related cultural and natural changes in China. Through cultivating wild and domesticated plants, recording the growth cycles and the impact of farming techniques, climate and other factors upon the plants, we can understand the influence of human interventions and natural factors such as temperature and precipitation upon the evolution of plants from wild to domesticated species. Further, we can also understand how plant cultivation might have changed human behaviour and culture, such as the occurrence of sedentism, the increased importance of farming in prehistoric economic structures, and the occurrence of property and land ownership. The soils and tools used in the cultivation experiments can also be analysed to provide reference data for the study of prehistoric farming techniques, use-wear analyses and prehistoric soil analysis, etc.

However, applying ancient techniques of wet rice cultivation it takes a long time for plants to show biological changes after being cultivated by humans, and a long time for the related environmental changes to take effect, experimentation and associated studies should be a continuing and long-term program. It also requires considerable resources, including funding, stable land, sufficient grains of wild plants, a laboratory to analyse phytolith and soil samples, and most importantly, researchers who have suitable training and can remain on the experimental land to continue the work for years, even decades. The aforementioned projects and findings are very preliminary as there have not been sufficient resources to facilitate long-term and ongoing cultivation experiments and associated projects.

Further, this integrated approach requires collaborations between scholars from different disciplines. It had been hoped that the chemical elements of soil used for cultivating different crops with different techniques could have been analysed in order to provide reference data for identifying prehistoric farming land, but this could not be achieved owing to the lack of expertise. Hopefully, more scholars will engage in cultivation experiments and associated initiatives in mainland China, and more data will be generated for an improved, in-depth and holistic understanding of the origin and development of agriculture in this region.

\section{Acknowledgements}

Harvesting and cultivation experiments, and the use-wear and phytolith analyses conducted by the author from 1999 to 2000 were funded by the French Fyssen Foundation, and the author is very grateful for the financial support she has received. The author would also like to thank Professor Fu Xianguo, Institute of Archaeology, Chinese Academy of Social Sciences, Professor Li Zhen, Archaeology Team of the Guangxi Zhuang Autonomous Region, and their team members for their continuous help and support in conducting various experiments in China from 1999 to 2012.

\section{References}

Anderson, P. (ed.). 1992. Préhistoire de l'Agriculture. Paris: CNRS Press.

Bellwood, P. 1978. Man's Conquest of the Pacific: The Prehistory of Southeast Asia and Oceania. Auckland: Collins.

— 2005. First Farmers: The Origins of Agricultural Societies. Malden, MA: Blackwell. 
Coles, J. 1979. Experimental Archaeology. New York: Academic Press.

Forrest, C. 2008. The nature of scientific experimentation in archaeology: Experimental archaeology from the nineteenth to the mid twentieth century. In P. Cunningham, J. Heeb and R. Paardekooper (eds), Experiencing Archaeology by Experiment, pp. 61-68. Oxford: Oxbow.

Harlan, J.R. 1967. A wild wheat harvest in Turkey. Archaeology 20(1): 197-201.

Hayden, B. 2003. Were luxury foods the first domesticates? Ethnoarchaeological perspectives from Southeast Asia. World Archaeology 34: 458-469. doi.org/10.1080/0043824021000026459a.

Hillman, G.C. and M.S. Davies. 1992. Domesticated rate in wild wheat and barley under primitive cultivation: preliminary results and archaeological implications of field measurements of selection coefficient. In P.C. Anderson (ed.), Préhistoire de l'Agriculture, pp. 113-148. Paris: CNRS Press.

Hu, L., Z. Chao, G. Min, F. Li, L. Chen, B. Liu, X. Li, Z. Huang, Y. Li, B. Xing and J. Dai. 2013. Evidence for a Neolithic Age fire-irrigation paddy cultivation system in the lower Yangtze River Delta, China. Journal of Archaeological Science 40(1): 72-78. doi.org/10.1016/j.jas.2012.04.021.

Lu, T.L.-D. 1998. Some botanical characteristics of green foxtail (Setaria viridis) and harvesting experiments on the grass. Antiquity 72(278): 902-907. doi.org/10.1017/S0003598X00087548.

—. 2002. A green foxtail (Setaria viridis) cultivation experiment in the middle Yellow River Valley and some related issues. Asian Perspectives 41: 1-14. doi.org/10.1353/asi.2002.0007.

- 2006. The occurrence of cereal cultivation in mainland China. Asian Perspectives 45: 129-158. doi.org/10.1353/asi.2006.0022.

- 2013. Daozuo yu Shiqian Wenhua Yanbian (Rice Farming and the Development of Prehistoric Culture). Beijing: Science Press (in Chinese).

Mathieu, J.R. 2002. Introduction. In J.R. Mathieu (ed.), Experimental Archaeology: Replicating Objects, Behaviors, and Processes, pp. 1-11. Oxford: BAR International Series 1035.

Oka, H.I. and W.T. Chang. 1959. The impact of cultivation on a population of wild rice, Oryza sativa f. spontanea. Phyton 13: 105-117.

Zheng, Y., G. Sun, L. Qin, C. Li, X. Wu and X. Chen. 2009. Rice fields and modes of rice cultivation between 5000 and 2500 BC in east China. Journal of Archaeological Science 36: 2609-2616. doi. org/10.1016/j.jas.2009.09.026. 
This text is taken from New Perspectives in Southeast Asian and Pacific Prehistory, edited by Philip J. Piper, Hirofumi Matsumura and David Bulbeck, published 2017 by ANU Press, The Australian National University, Canberra, Australia. 